\title{
Investigation of a Vertical Optical Coupler based on Trapezoidal grating for Symmetrical Coupling
}

\author{
Shadi A. Alboon ${ }^{1,2^{*}}$, Abdullah Karar ${ }^{1}$, Julien M. Barakat ${ }^{1}$ and Ibrahim Mahariq ${ }^{1}$ \\ ${ }^{1}$ College of Engineering and Technology, American University of the Middle East, Kuwait, \\ shadi.alboon@aum.edu.kw \\ ${ }^{2}$ Electronics Engineering Department, Hijjawi Faculty for Engineering, Yarmouk University, Jordan.
}

\begin{abstract}
Coupling light from a single-mode fiber into a waveguide is a significant issue. Numerous remedies have been introduced to this issue. In this paper, we numerically investigate a trapezoidal grating-based vertical coupler for symmetrical coupling applications. The power efficiency in both horizontal directions is identical. The coupling efficiency for the coupler is $48.46 \%$. Adding a reflection layer below the waveguide enhances the efficiency to $57.8 \%$. The coupler shows promising results for symmetrical coupling applications.
\end{abstract}

Key words: Optical coupler, trapezoidal grating, symmetrical coupling.

\section{INTRODUCTION}

Highlight Modern optics is one of the fields where basic physics is intimately related to technical applications. Nowadays there is a silent revolution in the optical components: freely propagating beams are being replaced by guided waves, and refractive lenses are being replaced by diffractive lenses incorporating grating which controls the light wave direction. The grating coupler, an essential device in integrated optics, is used to connect fibers and planar waveguides deprived of transitional optical devices.

Vertical coupling provides a dense optical connection and takes advantage of economies of scale since it needs a tiny space comparing to edge coupling. These new components allow for much higher degrees of integration and miniaturization. This approach is gaining more and more importance in telecommunication as well as in sensor technology since all the devices in these fields are in the micro or even the nano range. It is challenging to couple the light from optical fibers into waveguides due to the size disparity between fibers and waveguides. Since the waveguide core thickness is only $100-300 \mathrm{~nm}$ also the guided-mode is forcefully restricted in the core while the fiber core size is few micrometers.

Optical gratings couplers are used to overcome this challenge. Grating couplers have been broadly considered in literature [1] - [4]. One of the advantages of the grating couplers; that it can be positioned anyplace in the device and can simply be incorporated. In the grating-based couplers, the coupling occurs outside of the waveguide plane, which allows optical I/O's to inhabit any part of a planar lightwave circuit (PLC) rather than limited to the edge of a PLC.

One of the most attractive gratings profiles is the trapezoidal grating profile [5] - [9]. The fabrication of such a profile is less difficult than the perfect triangle profile [5]. Moreover, the trapezoidal profile reduces the fiber to fiber losses compared to the rectangular profile [6]. Diffraction grating with a trapezoidal profile shown advantages in many applications such as enhancing the efficiency in solar cells [7] - [8]. Furthermore, Micro-structured dielectric trapezoidal gratings are used in Terahertz Applications [9].

\section{OPTICAL COUPLER SIMULATION}

This section presents the simulation geometry of trapezoidal grating optical coupler. Also, it presents and discus the simulation results.

\subsection{Simulation Geometry}

Figure 1 shows the trapezoidal grating profile with different parameters. Such as, the grating period $(\Lambda)$, fill factor $(\mathrm{FF}=$ $d / \Lambda)$, depth $(h)$ and slope angles $\left(\theta_{1}\right.$ and $\left.\theta_{2}\right)$ of the gratings.

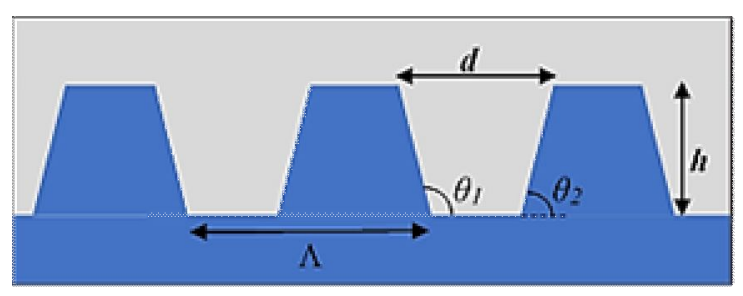

Figure 1: Trapezoidal grating profile 
Figure 2 shows the simulation geometry with the different components and parameters. An FDTD tool integrated with a micro genetic algorithm is used in the simulation and analysis of the coupler.

The following parameters were used in the simulation. The fiber core radius is $8.3 \mu \mathrm{m}$, $\mathrm{n} \_$core $=1.47$ and $\mathrm{n} \_$cladding $=$ 1.4647. The waveguide core is $n=3.4$ with a core thickness of $240 \mathrm{~nm}$. The waveguide upper and lower claddings are $\mathrm{n}=$ 1.46 and $n=1.444$ respectively. The thicknesses of the waveguide upper and lower cladding are $1.355 \mu \mathrm{m}$ and 1.005 $\mu \mathrm{m}$ respectively. The simulation region dimension is $32 \mu \mathrm{mX}$ $3 \mu \mathrm{m}$ with a Yee cell of (20nm x 20nm).

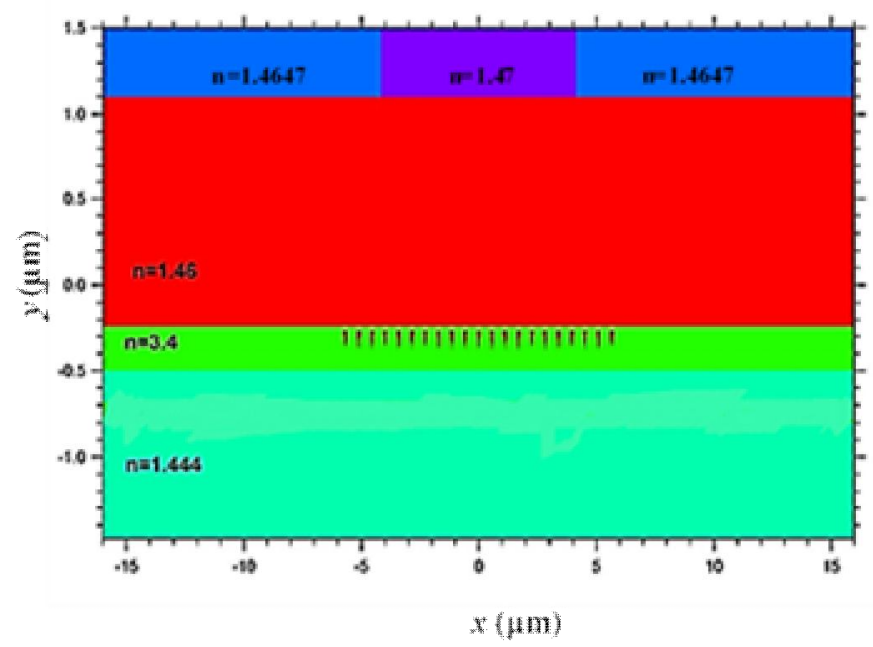

Figure 2: The simulation geometry under study.

\subsection{Simulation Results}

The optimized parameters of the trapezoidal gratings are: the period $(\Lambda=0.5662 \mu \mathrm{m})$, the fill factor $(\mathrm{FF}=d / \Lambda=0.292)$ and the grating depth $(h)$ is $215 \mathrm{~nm}$. The grating slope angels (see figure 1) are $\theta_{1}=125.26^{\circ}$ and $\theta_{2}=54.74^{\circ}$. The simulation results for the previous parameters are shown in figure 3 .

Figure 3 shows that most of the power launched from the fiber is coupled into the waveguide. Moreover, the figure shows that the coupling is symmetrical to the left and right of the waveguide ( $+x$ and $-x$-direction). Table 1 shows the power efficiency into the waveguide $(+x$ and $-x$-direction) and reflected and transmitted power $(+y$ and $-y$-direction respectively). The coupling efficiency is calculated using the mode overlap integral (MOI) [10].

As we can see from figure 3 and table 1 the coupler coupled then light in $+x$ and $-x$-directions, into the waveguide, symmetrically. Therefore, this coupler is useful in applications that required such coupling. Moreover, it can be used in measurement and test systems. Also, in optical communication and monitoring devices.

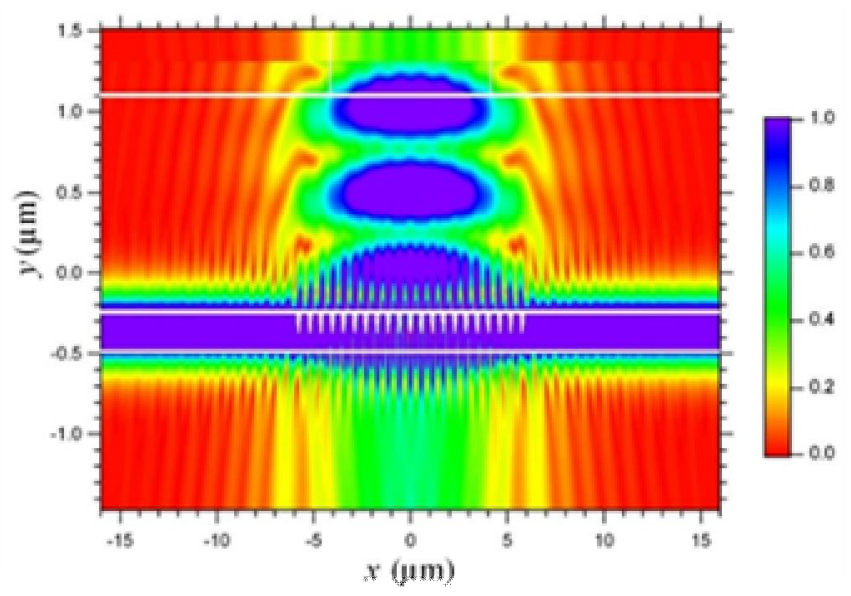

Figure 3: Plot of the field of the optimized grating structure.

Table 1 shows that more than $40 \%$ of the power is transmitted through or reflected from the waveguide core. In order to enhance the coupling efficiency into the waveguide, a reflection layer is added below the waveguide. This layer will reduce the transmitted power to the lower cladding. Therefore, it increases the coupling efficiency into the waveguide core.

Table 1: Power efficiency in the simulation geometry.

\begin{tabular}{|l|l|}
\hline Power Efficiency (+x) & $28.33 \%$ \\
\hline Power Efficiency (-x) & $28.33 \%$ \\
\hline Power Efficiency (+y) & $17.94 \%$ \\
\hline Power Efficiency (-y) & $25.00 \%$ \\
\hline Coupling Efficiency & $48.46 \%$ \\
\hline
\end{tabular}

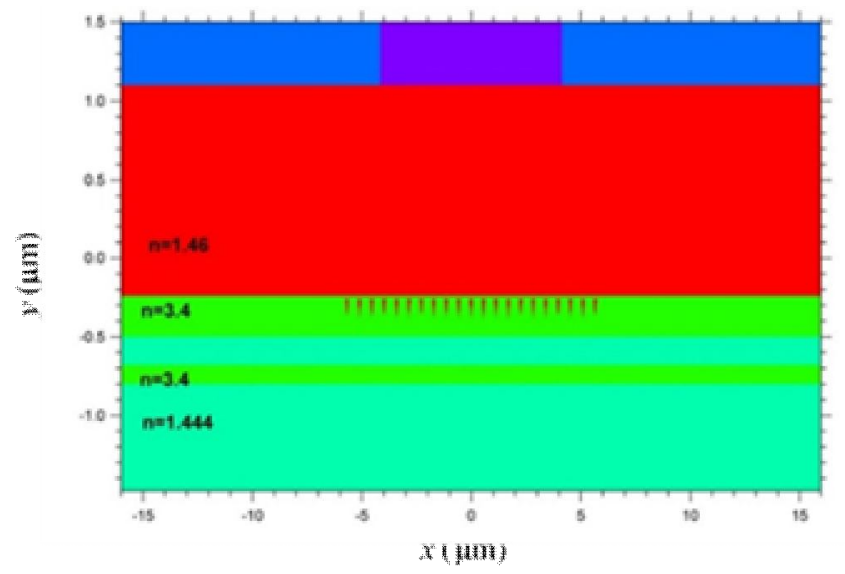

Figure 4: The simulation geometry with reflection layer added. 
Figure 4 shows the modified simulation geometry after added the reflection layer. The optimized parameters of the trapezoidal gratings are: the period $(\Lambda=0.5667 \mu \mathrm{m})$, the fill factor $(\mathrm{FF}=0.315)$ and the grating depth is $222 \mathrm{~nm}$. The grating angels (see figure 1) are $\theta_{1}=125.26^{\circ}$ degree $\theta_{2}=$ $54.74^{\circ}$. The optimized thickness of the reflection layer $(\mathrm{n}=3.4)$ is $102 \mathrm{~nm}$. While the vertical distance between the waveguide core and the refection layer is $180 \mathrm{~nm}$. The simulation results for the previous parameters are shown in figure 5. Table 2 shows the power efficiency in the modified geometry.

We can see from figure 5 and table 2 that the Si reflection layer reduces the transmitted power from $25 \%$ to almost $9 \%$. Moreover, the coupling efficiency is enhanced to $57.8 \%$.

Table 2: Power efficiency in the simulation modified geometry.

\begin{tabular}{|l|l|}
\hline Power Efficiency (+x) & $35.53 \%$ \\
\hline Power Efficiency (-x) & $35.53 \%$ \\
\hline Power Efficiency (+y) & $19.88 \%$ \\
\hline Power Efficiency (-y) & $8.8 \%$ \\
\hline Coupling Efficiency & $57.8 \%$ \\
\hline
\end{tabular}

While the power efficiency in both $x$ directions is increased to $35.53 \%$. that means adding the reflection layer enhances the power in the horizontal direction by $25.4 \%$. while enhancing the coupling efficiency by $19.3 \%$.

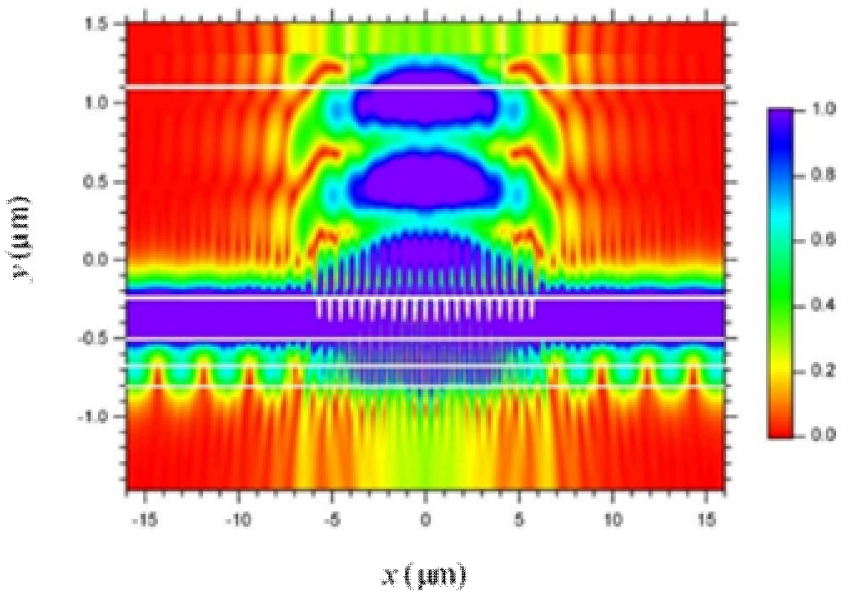

Figure 5: Plot of the field of the optimized grating structure.

\section{CONCLUSION}

This paper discusses the design and analysis of dielectric trapezoidal grating coupler for symmetrical coupling. The simulation results show that the power coupling efficiency is the same for both $-x$ and $+x$-direction. The optimized values of the gratings provide a $48.46 \%$ coupling efficiency. In order to enhance this efficiency, a reflection layer is added to increase the coupling efficiency to $57.8 \%$. Such coupler is appropriate for the testing of photonic devices. This coupler opens the door for new applications in the sensing based on the symmetrical coupling.

Please note that the references at the end of this document are in the preferred referencing style. Give all authors' names; do not use "et al." unless there are six authors or more. Use a space after authors' initials. Papers that have not been published should be cited as "unpublished" [4]. Papers that have been submitted for publication should be cited as "submitted for publication" [5]. Papers that have been accepted for publication, but not yet specified for an issue should be cited as "to be published" [6]. Please give affiliations and addresses for private communications [7].

Capitalize only the first word in a paper title, except for proper nouns and element symbols. For papers published in translation journals, please give the English citation first, followed by the original foreign-language citation [8].

\section{REFERENCES}

1. M. Dai, L. Ma, Y. Xu, M. Lu, X. Liu, and Y. Chen. Highly efficient and perfectly vertical chip-to fiber dual-layer grating coupler, Optics Express 23 (2), pp. 1691-1698, 2015.

https://doi.org/10.1364/OE.23.001691

2. Q. Liu and W. Wang. Free-standing GaN grating couplers and rib waveguide for planar photonics at telecommunication wavelength, Optics \& Laser Technology, 98, pp. 257-263, 2018.

3. Z. Zhang, et al. Highly efficient vertical fiber interfacing grating coupler with bilayer antireflection cladding and backside metal mirror, Optics \& Laser Technology, 90, pp. 136-143, 2017.

4. $\mathrm{H}$. Li, et al. Investigation of the chip to photodetector coupler with subwavelength grating on SOI, Optics \& Laser Technology, 76, pp. 79-84, 2016.

5. H. Guan, Z. Chen, Y. Jin, K. Yi, and J. Shao. An effective design method for trapezoidal pulse compression metal multilayer dielectric gratings, in proc. International Conference on Numerical Simulation of Optoelectronic Devices (NUSOD), 2015, pp. 165-166.

6. L. R. Chen. Subwavelength grating waveguide devices in silicon on-insulators for integrated microwave photonics, Chinese Optics Letters, 15 (1), pp. 010004-1- 010004-5, 2017.

7. M. Dehdast, A. Bahrami and S. Mohammadnejad. A novel trapezoidal profile of optimized diffraction grating for light trapping in thin silicon solar cells, Optica Applicata, XLVII (1), pp. 75-83, 2017.

8. N. Das and S. Islam. Design and Analysis of Nano-Structured Gratings for Conversion Efficiency Improvement in GaAs Solar Cells, Energies, 9 (690), pp. 1-13, 2016. 
Shadi A. Alboon et al., International Journal of Emerging Trends in Engineering Research, 8(2), February 2020, 277 - 280

9. M. Hajji, D. Zeze, C. Balocco and A. J. Gallant. Artificial Microstructured Dielectric Layers for Terahertz Applications, in proc. 4lst International Conference on Infrared, Millimeter, and Terahertz waves (IRMMW-THz), 2016, pp. 1-2.

10. M. Stallein, C. Kolleck, and G. Mrozynski. Improved Analysis of the Coupling of Optical Waves into Multimode Waveguides Using Overlap Integrals, Progress In Electromagnetics Research Symposium, 2005, pp. 464-468. 